\title{
UNA POBLACIÓN RELICTA DE ACER SACCHARUM SUBSP. SKUTCHII (ACEROIDEAE) EN EL ESTADO DE GUERRERO, MÉXICO
}

\author{
YaLma L. VARGas-RodRigueZ \\ Louisiana State University, 107 Life Sciences Building, Department of Biological \\ Sciences, Baton Rouge 70803, Louisiana, U.S.A., yvarga1@tigers.lsu.edu
}

\section{RESUMEN}

Sedescribela estructura, composición, diversidad, relaciones ambientales y conservación de un bosque mesófilo con Acer saccharum subsp. skutchii (arce azucarero) recientemente encontrado en el municipio de Coahuayutla de José María Izazaga, Guerrero. Se registraron los individuos leñosos con $\geq 1 \mathrm{~cm}$ dap en parcelas circulares de $10 \mathrm{~m}$ de radio, y la altura de plántulas y plantas juveniles del arce azucarero en parcelas de $5.6 \mathrm{~m}$ de radio. Para cada especie se obtuvo su valor de importancia. Se utilizó la técnica de ordenación Escalar Multidimensional No-métrica (NMDS) para explorar sus correlaciones con variables edafológicas, coordenadas geográficas, de elevación y apertura de claros. Se registraron 43 especies de plantas leñosas en una superficie de 0.126 ha. Las familias mejor representadas fueron Asteraceae y Fagaceae. La distribución diamétrica de las plantas leñosas mostró una forma de “J” invertida, indicando regeneración exitosa. El arce azucarero fue codominante en el dosel con Carpinus caroliniana y Quercus uxoris. Tanto en la población de Guerrero, como en las otras cinco localidades donde habita el árbol en México y Guatemala, la mayoría de las correlaciones encontradas entre la presencia y ausencia de árboles, incluyendo Acer saccharum subsp. skutchii, y los factores ambientales se refirieron a las características de suelo. De las especies registradas en el estudio, 30\% están incluidas en alguna categoría de riesgo. El régimen de incendios en la zona pone en peligro la persistencia de este árbol en el sitio de estudio.

Palabras clave: Acer saccharum, bosque mesófilo de montaña, especies disyuntas, fitogeografía, gradientes ambientales, Guerrero, México.

\section{ABSTRACT}

Structure, composition, diversity, environmental relationships, and conservation status of a cloud forest with Acer saccharum subsp. skutchii (sugar maple) are described 
in a recently found locality at Coahuayutla de José María Izazaga municipality, state of Guerrero, Mexico. Woody individuals $\geq 1 \mathrm{~cm}$ dbh in $10 \mathrm{~m} /$ radius circular plots and seedlings and saplings heights of sugar maple in $5.6 \mathrm{~m} /$ radius circular plots were recorded. The relative ecological importance of each species was expressed in an importance value. Non-metric Multidimensional Scaling (NMDS) ordination technique was used to explore the species correlations with edaphic, geographic coordinates, elevation, and canopy gaps variables. 43 woody species in 0.126 ha were recorded. The families with most species were Asteraceae and Fagaceae. The diametric distribution of woody species showed an inverted "J" shape, indicating successful regeneration. Sugar maple is co-dominant with Carpinus caroliniana and Quercus uxoris. Most correlations between presence-absence of trees, including Acer saccharum subsp. skutchii, were with soil characteristics in the population of Guerrero and all maple localities in Mexico and Guatemala. 30\% of the reported species are included in some risk category. The fire regime in the zone is a major threat for the persistence of the studied species.

Key words: Acer saccharum, cloud forest, disjunct species, environmental gradients, Guerrero, Mexico, phytogeography.

\section{INTRODUCCIÓN}

Los arces ("maples") pertenecen al género Acer, subfamilia Aceroideae, familia Sapindaceae (APG, 1998; 2003). Son de distribución holártica y habitan en bosques mesófilos en México y Centroamérica (Graham, 1999; Qian y Ricklefs, 2000). Con base en el registro paleobotánico, se sugiere que el área actual de las especies de este tipo de afinidad en nuestro país es el resultado de eventos geológicos y climáticos ocurridos durante el Terciario y el Pleistoceno, que restringieron la distribución de esas plantas a las barrancas húmedas ubicadas en elevaciones entre 1200 y 2500 m (Martin y Harrell, 1957; Graham, 1999). En México, los bosques mesófilos en los que habitan algunas de las especies de esta vinculación geográfica, comparten un número significativo de géneros disyuntos con el este de Asia (Qian y Ricklefs, 2000; Vargas-Rodriguez, 2005).

La presencia de Acer más al sur de Estados Unidos fue reconocida por Rehder (1936), quien describió a Acer skutchii de Guatemala. El taxon fue subsecuentemente reclasificado como una subespecie (Acer saccharum subsp. skutchii (Rehder) Murray) del arce norteamericano A. saccharum (Murray, 1975). En Guatemala, las poblaciones de arces se localizan en los departamentos de El Quiché (Sierra de los 
Cuchumatanes), Zacapa y El Progreso (Sierra de Minas) (Rehder, 1936; MedinillaSánchez, 1999; Anónimo, 2005; Vargas-Rodriguez 2005). En México, el primer registro de este taxon fue de Tamaulipas (Hernández-Xolocotzi et al., 1951). Con excepción de la localidad en este último estado, la subespecie no es fácil de observar en nuestro país. Por ejemplo, una población de Chiapas fue explorada inicialmente en 1953 por F. Miranda (Breedlove, 1986) y la siguiente colecta se realizó en el año 2003 en el mismo sitio por Vargas-Rodriguez (Vargas-Rodriguez, 2005). Recientemente se encontraron otros dos rodales en el occidente de México (Jardel et al., 1996; Vázquez-García et al., 2000).

El límite sur de la distribución de los arces azucareros en América se registra a la latitud $15^{\circ}$ N. A. saccharum subsp. skutchii es una planta de bosque mesófilo, relicta, de repartición disyunta e incluida en la Norma Oficial Mexicana (NOM-059 -ECOL-2001) para su protección (Anónimo, 2002a). Los bosques mesófilos son el hábitat favorable para su existencia, así como para la de muchas otras especies con afinidad holártica, sin embargo, estas comunidades vegetales tienen una superficie reducida en el territorio mexicano y están seriamente afectadas por el disturbio antrópico.

En el estado de Guerrero se registró la presencia de arce azucarero a través de la colecta de $R$. Mayorga 1519 (IEB), realizada en el año 2000. Sin embargo, debido a que tal material tardó en incorporarse al acervo de herbarios, la localidad no fue incluida en un trabajo sintético realizado sobre las poblaciones de esta planta en México (Vargas-Rodriguez, 2005). El objetivo de la presente contribución es el de describir las características estructurales, la composición y diversidad del bosque mesófilo que habita, la relación de la planta y de árboles coexistentes con las variables ambientales y, finalmente, definir el estado de conservación del arce azucarero en el estado de Guerrero. Las características de este rodal se ubican después en el contexto de las demás poblaciones conocidas de la subespecie en México y Guatemala.

\section{MÉTODOS}

La población estudiada se encuentra en la barranca de El Silencio, al este de la localidad de Primer Campo, ejido de El Aguacate, al noreste de Coahuayutla, municipio de Coahuayutla de José María Izazaga. El sitio forma parte de la Sierra Cucharillos, en las coordenadas $18^{\circ} 11^{\prime} \mathrm{N}$ y 101 $24^{\prime}$ W, con una elevación de $2110 \mathrm{~m}$ (Fig. 1). De acuerdo con la carta climática, escala 1:1'000,000 (se carece de carta con escala menor para el sitio), el clima es de tipo $\mathrm{C}\left(\mathrm{w}_{2}\right) \mathrm{w}$, templado subhúmedo, 

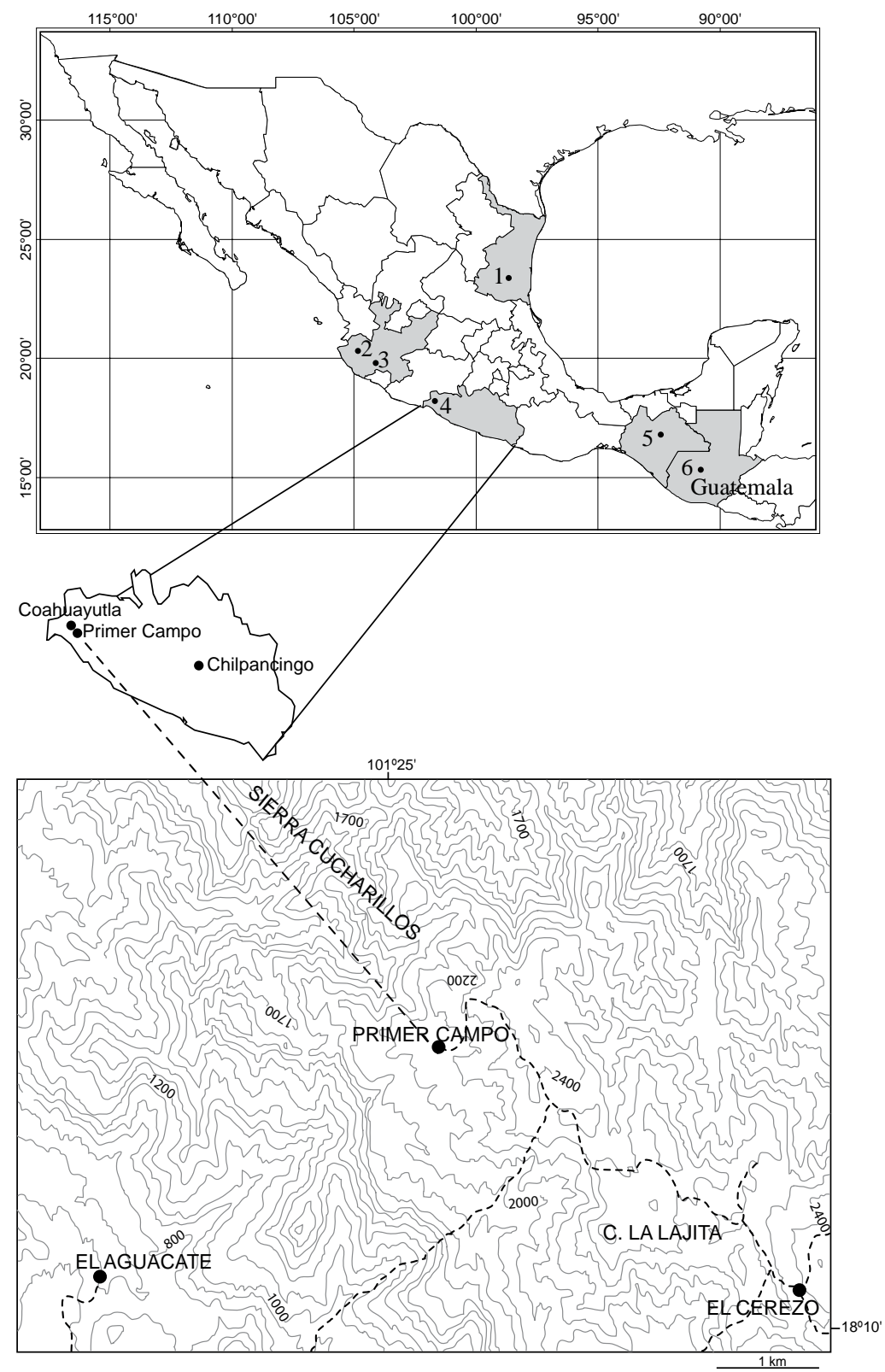

Fig. 1. Ubicación de la población de Acer saccharum subsp. skutchii en Guerrero. La localización de otras poblaciones de la subespecie en México y Guatemala se señalan con número: 1) Reserva de la Biosfera El Cielo, Tamaulipas, 2) Talpa de Allende, Jalisco, 3) Reserva de la Biosfera Sierra de Manantlán, Jalisco, 4) Barranca El Silencio, Guerrero, 5) Tenejapa, Chiapas, 6) Reserva de la Biosfera Sierra de las Minas, Guatemala. 
con porcentaje de lluvia invernal $<5$ y la precipitación del mes más seco de $<40 \mathrm{~mm}$ (Anónimo, 1981). No existen datos precisos de temperatura y precipitación para este sitio. Los suelos predominantes son de los tipos regosol éutrico, luvisol crómico, litosol; de clase textural media y derivados de rocas ígneas extrusivas del Terciario Superior (Anónimo, 1983a; 1983b).

En la localidad se ubicaron cuatro círculos de $10 \mathrm{~m}$ de radio cada uno, con lo cual el área total muestreada fue 0.126 ha. La cañada donde habita la especie es estrecha y está rodeada de bosque de pino afectado por un incendio reciente, por lo que el número y ubicación de los círculos dependió de la densidad del arce azucarero y el tamaño de la superficie del bosque remanente que habita. El muestreo se realizó en el mes de octubre de 2006.

En cada círculo se registraron las especies de árboles y/o arbustos enraizados dentro del mismo. Se midieron los diámetros a la altura de $1.3 \mathrm{~m}$ (dap) de las plantas leñosas $\geq 1 \mathrm{~cm}$ (dap). También se registraron las alturas de plántulas (individuos $\leq 130$ $\mathrm{cm}$ de altura) e individuos juveniles ( $<1 \mathrm{~cm}$ dap y $\geq 130 \mathrm{~cm}$ de altura) de arce azucarero en un círculo interior de $5.64 \mathrm{~m}$ de radio, concéntrico con el principal de $10 \mathrm{~m}$. El criterio utilizado para el registro de alturas siguió a Olvera-Vargas et al. (1996) y Figueroa-Rangel y Olvera-Vargas (2000). Se calculó el porcentaje de individuos $\geq 1$ cm por categoría diamétrica y el porcentaje de individuos de plántulas e individuos juveniles por categoría de alturas. Estos últimos valores se graficaron y se analizó si las distribuciones obtenidas semejaban una J-invertida.

Se colectó cuando menos un ejemplar de cada especie de árbol o arbusto presente dentro de los círculos. Su determinación se realizó por los especialistas del Instituto de Botánica (Herbario IBUG) y del Departamento de Geografía de la Universidad de Guadalajara. Los especímenes se depositaron en el herbario IBUG de la Universidad de Guadalajara y en el herbario LSU de la Universidad Estatal de Louisiana. La nomenclatura siguió el International Plant Name Index (IPNI, 1999).

Se registraron las condiciones de sitio en la población de arce azucarero. La ubicación geográfica y elevación se determinaron usando un geoposicionador (GPS12 Garmin-Corporation) y la exposición y pendiente usando un compás y un clinómetro, respectivamente. Se tomó una medida de apertura del dosel en el centro de cada círculo usando un densiómetro y se hicieron observaciones de presencia e impacto de ganado e incendios.

Se tomó una muestra de suelo (30 cm de profundidad) en cada círculo; éstas se analizaron en el Centro de Investigación y Desarrollo Industrial de la Universidad Autónoma de Guadalajara. Se determinó el pH, conductividad eléctrica, textura, 
materia orgánica, nutrimentos (nitrógeno nítrico, nitrógeno amoniacal, $\mathrm{P}, \mathrm{K}, \mathrm{Ca}, \mathrm{Cu}$, $\mathrm{Mg}, \mathrm{Mn}, \mathrm{Na}, \mathrm{Fe}, \mathrm{S}, \mathrm{Zn}$, sulfatos), capacidad de intercambio catiónico, densidad aparente y humedad del suelo. El análisis de los datos anteriores se realizó de acuerdo con lo establecido en la Norma Oficial Mexicana (Anónimo, 2002b).

La importancia ecológica relativa de cada especie leñosa encontrada se expresó en un índice de valor de importancia (IVI), que se obtuvo promediando los valores relativos de dominancia, densidad de tallos y frecuencia (Curtis y McIntosh, 1951). Se determinó la diversidad (H’) y uniformidad (E') con el índice de ShannonWiener usando el logaritmo de base e (Magurran, 1988; McCune y Grace, 2002). El software utilizado para estos cálculos fue PC-ORD v4.27 (McCune y Mefford, 1999).

Para cada uno de los cuatro círculos se elaboró una matriz de datos de presencia y ausencia de especies leñosas y otra con los valores de variables ambientales (elevación, latitud, longitud, apertura de dosel, pendiente y características de suelo, omitiéndose los de temperatura y precipitación ya que no estuvieron disponibles para todos los sitios). Se incluyeron además en dichas matrices los mismos datos correspondientes a las otras cinco poblaciones conocidas (en las Reservas de la Biosfera El Cielo, Sierra de Manantlán, Sierra de las Minas y en los municipios de Talpa de Allende y Tenejapa) de Acer saccharum subsp. skutchii con la finalidad de obtener patrones de correlación más sólidos. Los valores de tales sitios fueron obtenidos de Vargas-Rodriguez (2005). El total de círculos analizados de las seis localidades fueron 57, y 28 variables ambientales. Se utilizó el procedimiento de ordenación Escalar Multidimensional No-métrica (NMDS), de acuerdo con el método descrito por Kruskal (1964) y Mather (1976). Esta técnica es efectiva en el análisis de comunidades, ya que no implica una relación lineal entre las variables (Minchin, 1987) y busca la mejor ubicación de $n$ entidades en $k$ ejes con la finalidad de reducir el estrés en la configuración $k$-dimensional (Minchin, 1987; McCune y Grace, 2002). Se usó una configuración de 1,500 repeticiones con datos reales para estimar estabilidad. Se evaluó la dimensionalidad de los datos empleando tres ejes en la ordenación y la probabilidad de que la ordenación tridimensional alcanzara un estrés final que fuera obtenido por el azar con la prueba de Monte Carlo con 1,500 repeticiones en datos reales y 900 con datos al azar. Se usó la distancia Sørensen en dicho análisis, ya que es sólida con datos ecológicos (Beals, 1984; Faith et al., 1987) y el coeficiente de correlación de Pearson para evaluar la relación entre los ejes identificados de la ordenación y las variables ambientales. El software utilizado para el análisis fue PC-ORD v4.27 (McCune y Mefford, 1999). 
Vargas-Rodríguez: Población relicta de Acer saccharum subsp. skutchii en Guerrero, México

\section{RESULTADOS}

En el bosque mesófilo con arce azucarero de la barranca El Silencio se encontraron 43 especies de plantas leñosas pertenecientes a 36 géneros y 30 familias; de estas últimas, las mejor representadas fueron Asteraceae (con cinco especies), Fagaceae (con tres), Araliaceae, Cornaceae, Lauraceae, Myrsinaceae, Pinaceae y Solanaceae, con dos cada una. La diversidad del sitio fue de $\mathrm{H}^{\prime}=1.641$ y la uniformidad de $\mathrm{E}^{\prime}=0.542$.

La densidad de individuos leñosos $\geq 1 \mathrm{~cm}$ dap fue de $2986 \pm 207$ ind. ha ${ }^{-1}$. El área basal de todos los árboles fue de $24.371 \mathrm{~m}^{2}$ por hectárea y fue menor que el registrado en las otras cinco localidades comparadas $\left(25.7\right.$ a $\left.52.2 \mathrm{~m}^{2} \mathrm{ha}^{-1}\right)$. Los arces azucareros constituyeron $27.7 \%$ del área basal total en la localidad de Guerrero y su densidad fue de $111 \pm 21$ ind. ha ${ }^{-1}$.

La distribución diamétrica de las especies leñosas presentó una forma de “J” invertida, con mayor cantidad de individuos en las categorías de diámetro pequeñas. Los árboles de talla más grande midieron hasta $80 \mathrm{~cm}$ dap y pertenecieron a Quercus uxoris. Acer saccharum subsp. skutchii alcanzó un máximo de $55 \mathrm{~cm}$ dap (Fig. 2).

La regeneración natural de Acer saccharum subsp. skutchii se compuso principalmente de individuos de 20 a $25 \mathrm{~cm}$ de altura. El total de plántulas de arce azucarero fue de 234 y de jóvenes uno (Fig. 2). La distribución de tamaños de ambos grupos semejó una forma de campana (Fig. 2).

Las especies con mayor IVI en la localidad de Guerrero fueron Carpinus caroliniana (12.17), Acer saccharum subsp. skutchii (12.09) y Quercus uxoris (11.99) (Cuadro 1). Cornus excelsa (0.49) y Dendropanax arboreus (0.49) estuvieron entre los árboles con menor valor (Cuadro 1).

La ordenación Escalar Multidimensional No-métrico con la prueba de Monte Carlo indicó un modelo tridimensional. El estrés final fue de 24.14, con la reducción del mismo en los ejes de 0.001 y la inestabilidad de 0.05268 con 500 iteraciones. La mayor parte del estrés se redujo después de 37 repeticiones.

La varianza en el primer eje fue explicada por una variable de suelo, elevación y latitud. En el eje dos lo fue por cinco valores de suelo y longitud geográfica. En el tres la varianza no se explicó por ningún valor (Cuadro 2, Fig. 3).

Aunque la variación en los ejes de la ordenación fue explicada por valores geográficos, de elevación y de suelo, las correlaciones más altas entre la presencia y ausencia de árboles y datos ambientales atañeron a las características de suelo. Tomando en cuenta todas las especies leñosas que habitan en los seis sitios que se com- 

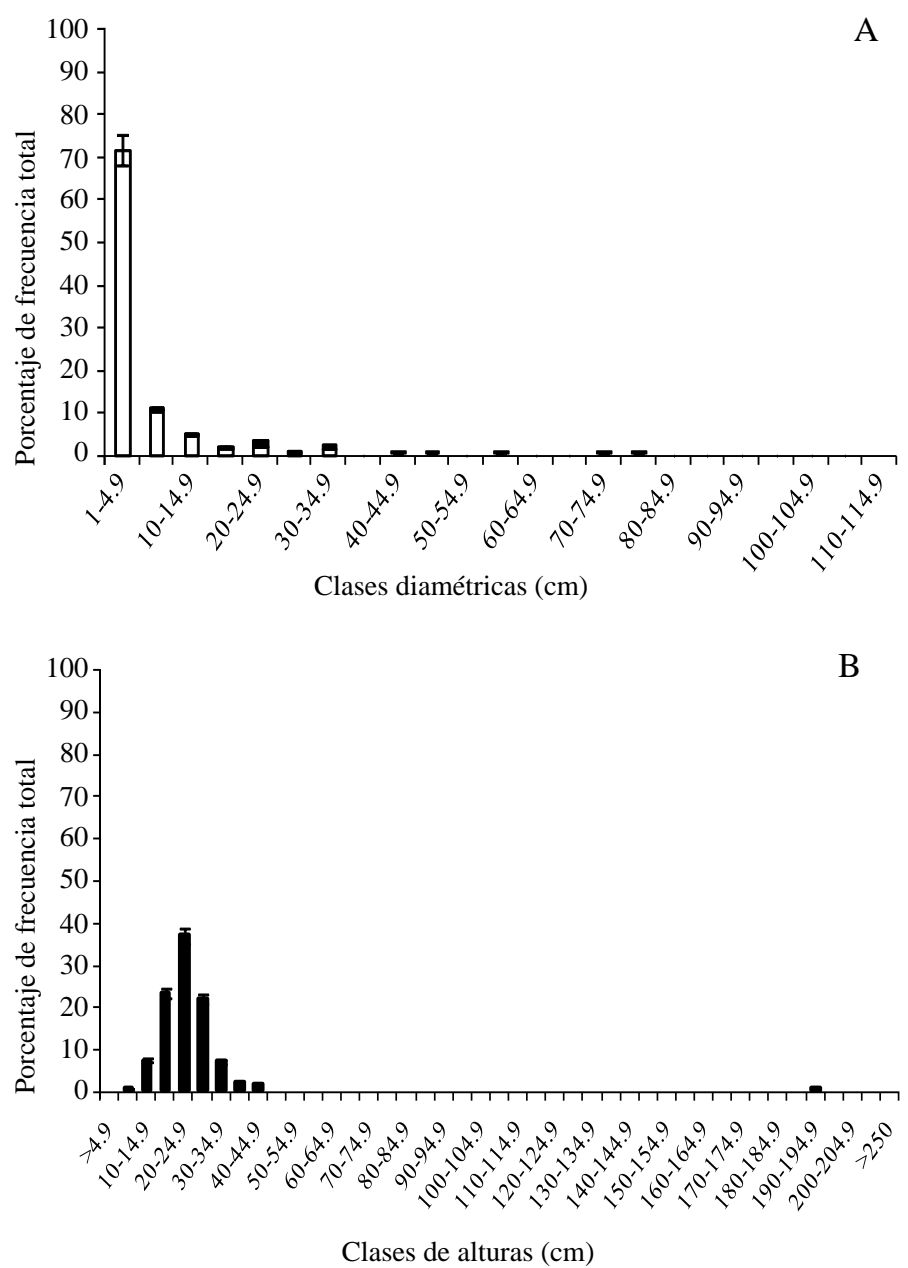

Fig. 2. A) Porcentaje de frecuencia total de árboles por categoría diamétrica; la sección en negro de cada barra indica la proporción de Acer saccharum subsp. skutchii. B) Porcentaje de frecuencia total de plántulas e individuos juveniles de arce por categoría de altura.

pararon donde crece el arce, la presencia de árboles se correlacionó positivamente con la capacidad de intercambio catiónico $(\mathrm{r}=0.618)$, la densidad aparente del suelo $(\mathrm{r}=0.693), \mathrm{Mg}(\mathrm{r}=0.511)$ y sulfatos $(\mathrm{r}=0.497)$ (Cuadro 2$)$. El diagrama de ordenación mostró una dispersión de los sitios de bosque mesófilo con arce de Guerrero, Chiapas y Guatemala a lo largo del eje 2, representado principalmente por los valores anteriormente mencionados (Fig. 3). 
Cuadro 1. Valor de importancia de cada especie registrada en el bosque mesófilo con arce azucarero de Guerrero. Superficie total 0.126 ha. Se incluyen los montos de densidad y área basal por especie.

\begin{tabular}{|c|c|c|c|c|}
\hline Familia & Especie & $\begin{array}{c}\text { Valor de } \\
\text { importancia } \\
(\%)\end{array}$ & $\begin{array}{l}\text { Densidad de } \\
\text { individuos/ha }\end{array}$ & $\begin{array}{c}\text { Área basal } \\
\mathrm{dm}^{2} / \mathrm{ha}\end{array}$ \\
\hline Betulaceae & Carpinus caroliniana Walter & 12.17 & 429.94 & 421.5 \\
\hline Sapindaceae & $\begin{array}{l}\text { Acer saccharum Marsh. subsp. } \\
\text { skutchii (Rehder) E. Murray }\end{array}$ & 12.09 & 111.46 & 675.67 \\
\hline Fagaceae & Quercus uxoris McVaugh & 11.99 & 159.24 & 629.22 \\
\hline Pinaceae & Pinus pseudostrobus Lindl. & 5.28 & 310.51 & 44.66 \\
\hline Asteraceae & Eupatorium sp.1 & 4.35 & 230.89 & 70.5 \\
\hline Myrsinaceae & Parathesis villosa Lundell & 4.21 & 246.82 & 18.09 \\
\hline Actinidiaceae & Saurauia serrata DC. & 4.05 & 191.08 & 22.91 \\
\hline Asteraceae & $\begin{array}{l}\text { Ageratina areolaris (DC.) Gage ex } \\
\text { B. L. Turner }\end{array}$ & 3.29 & 207.01 & 12.91 \\
\hline Myrsinaceae & Synardisia venosa (Mast.) Lundell & 3.29 & 111.46 & 31.85 \\
\hline Cornaceae & $\begin{array}{l}\text { Cornus disciflora Sessé \& Moc. ex } \\
\text { DC. }\end{array}$ & 3.01 & 31.85 & 105.98 \\
\hline Pinaceae & $\begin{array}{l}\text { Abies guatemalensis Rehder var. } \\
\text { longibracteata Z. Debreczy \& I. } \\
\text { Rácz }\end{array}$ & 2.91 & 23.89 & 134.17 \\
\hline Asteraceae & Senecio angulifolius DC. & 2.88 & 111.46 & 1.87 \\
\hline Magnoliaceae & Magnolia schiedeana Schltdt. & 2.74 & 87.58 & 40.47 \\
\hline Solanaceae & Cestrum aurantiacum Lindl. & 2.14 & 79.62 & 3.15 \\
\hline Theaceae & Cleyera velutina B. M. Barthol. & 2.1 & 39.81 & 62.56 \\
\hline Symplocaceae & Symplocos citrea Lex. & 2.02 & 95.54 & 10.95 \\
\hline Rhamnaceae & $\begin{array}{l}\text { Rhamnus hintonii M. C. Johnst. \& } \\
\text { L. A. Johnst. }\end{array}$ & 1.55 & 55.73 & 9.09 \\
\hline Aquifoliaceae & Ilex brandegeeana Loes. & 1.43 & 47.77 & 6.64 \\
\hline Fagaceae & Quercus acutifolia Née & 1.39 & 7.96 & 65.53 \\
\hline Polygalaceae & Monnina sylvatica Schltdl. \& Cham. & 1.27 & 39.81 & 1.34 \\
\hline Lauraceae & Persea chrysantha Lorea-Hern. & 1.23 & 23.89 & 11.72 \\
\hline Styracaceae & Styrax argenteus C. Presl & 1.23 & 23.89 & 11.47 \\
\hline Melastomataceae & $\begin{array}{l}\text { Conostegia volcanalis Standl. \& } \\
\text { Steyerm. }\end{array}$ & 1.21 & 31.85 & 3.9 \\
\hline Celastraceae & Zinowiewia concinna Lundell & 1.03 & 15.92 & 3.3 \\
\hline Salicaceae & Populus simaroa Rzed. & 0.96 & 15.92 & 27.78 \\
\hline
\end{tabular}


Continuación. Cuadro 1.

\begin{tabular}{|c|c|c|c|c|}
\hline Familia & Especie & $\begin{array}{l}\text { Valor de } \\
\text { importancia } \\
\text { (\%) }\end{array}$ & $\begin{array}{l}\text { Densidad de } \\
\text { individuos/ha }\end{array}$ & $\begin{array}{c}\text { Área basal } \\
\mathrm{dm}^{2} / \mathrm{ha}\end{array}$ \\
\hline Clethraceae & $\begin{array}{l}\text { Clethra lanata M. Martens \& } \\
\text { Galeotti }\end{array}$ & 0.86 & 39.81 & 1.35 \\
\hline Onagraceae & Fuchsia microphylla Kunth & 0.86 & 39.81 & 0.86 \\
\hline Flacourtiaceae & Xylosma flexuosum (Kunth) Hemsl. & 0.68 & 23.89 & 0.59 \\
\hline Simaroubaceae & $\begin{array}{l}\text { Picramnia guerrerensis W. W. } \\
\text { Thomas }\end{array}$ & 0.6 & 15.92 & 1.4 \\
\hline Caesalpiniaceae & $\begin{array}{l}\text { Senna multifoliolata (Paul G. } \\
\text { Wilson) H. S. Irwin \& Barneby var. } \\
\text { metaxi H. S. Irwin \& Barneby }\end{array}$ & 0.59 & 15.92 & 0.6 \\
\hline Fagaceae & Quercus martinezii C. H. Müll. & 0.59 & 15.92 & 0.72 \\
\hline Leguminosae & Calliandra sp. & 0.59 & 15.92 & 0.51 \\
\hline Araliaceae & $\begin{array}{l}\text { Oreopanax xalapensis (Kunth) } \\
\text { Decne. \& Planch. }\end{array}$ & 0.5 & 7.96 & 0.37 \\
\hline Asteraceae & Baccharis conferta Kunth & 0.5 & 7.96 & 0.52 \\
\hline Asteraceae & Eupatorium sp.2 & 0.5 & 7.96 & 0.36 \\
\hline Fabaceae & $\begin{array}{l}\text { Desmodium pseudo-amplifolium } \\
\text { Micheli }\end{array}$ & 0.5 & 7.96 & 0.61 \\
\hline Lamiaceae & Salvia mexicana $\mathrm{L}$ & 0.5 & 7.96 & 0.39 \\
\hline Lauraceae & Persea donnell-smithii Mez & 0.5 & 7.96 & 0.51 \\
\hline Phyllonomaceae & Phyllonoma laticuspis Engl. & 0.5 & 7.96 & 0.46 \\
\hline Amaranthaceae & $\begin{array}{l}\text { Iresine aff. diffusa Humb. \& Bonpl. } \\
\text { ex Willd. }\end{array}$ & 0.49 & 7.96 & 0.26 \\
\hline Araliaceae & $\begin{array}{l}\text { Dendropanax arboreus (L.) Decne. } \\
\text { \& Planch. }\end{array}$ & 0.49 & 7.96 & 0.1 \\
\hline Cornaceae & Cornus excelsa Kunth & 0.49 & 7.96 & 0.21 \\
\hline Solanaceae & Cestrum aff. oblongifolium Schltdl. & 0.49 & 7.96 & 0.07 \\
\hline
\end{tabular}

En lo que concierne a las plantas incluidas en alguna categoría de protección de la Lista Roja de la Unión Internacional para la Conservación de la Naturaleza y en la Norma Ecológica NOM-059-ECOL-2001 (Anónimo, 2001; Anónimo, 2002a) son 12 (28\%); solamente Magnolia schiedeana y Saurauia serrata están en ambas. Por su parte, en la Lista Roja de la UICN con la categoría en peligro se registran Magnolia schiedeana y Saurauia serrata; como vulnerables a Cornus disciflora y 
Cuadro 2. Coeficientes de correlación de Pearson (r) entre variables ambientales y los ejes de ordenación usando valores de presencia y ausencia de árboles de las seis localidades de Acer saccharum subsp. skutchii. Grados de libertad son 26, valor de significancia $\mathrm{p}<0.01$.

\begin{tabular}{|c|c|c|c|}
\hline \multicolumn{2}{|r|}{ Variable } & \multicolumn{2}{|c|}{ Ejes } \\
\hline Suelo & & 1 & 2 \\
\hline & Capacidad de intercambio catiónico & & 0.618 \\
\hline & Conductividad eléctrica & -0.525 & \\
\hline & Densidad aparente & & 0.693 \\
\hline & $\mathrm{Mg}$ & & 0.511 \\
\hline & $\mathrm{Na}$ & & -0.501 \\
\hline & Sulfatos & & 0.497 \\
\hline
\end{tabular}

Geográficas

\begin{tabular}{lr} 
Elevación & -0.478 \\
Latitud & 0.586 \\
Longitud & \\
\hline
\end{tabular}
$-0.665$

Quercus uxoris; como preocupación menor a Pinus pseudostrobus y Quercus martinezii. En la NOM-059-ECOL-2001 se consideran como en peligro de extinción a Abies guatemalensis, Acer skutchii y Zinowiewia concinna; como amenazadas a Carpinus caroliniana y Magnolia schiedeana; y como protección especial a Populus simaroa y S. serrata.

Es posible que las plantas de la región de estudio identificadas como Magnolia schiedeana en realidad corresponden a Magnolia guerrerensis; sin embargo, los pocos ejemplares colectados en la barranca El Silencio tuvieron flores inmaduras, lo que dificultó la determinación exacta y no se pudo concluir que se tratase de la última. M. guerrerensis es una especie dada a conocer recientemente, endémica del centro de Guerrero (municipios de Leonardo Bravo, Heliodoro Castillo y Quechultenango), de abundancia baja, reducido número de localidades y con grado elevado de amenaza antrópica. Dado que su descripción es reciente (Jiménez-Ramírez et al., 2007), no se ha considerado en la Lista Roja de la UICN o en la Norma Oficial Mexicana para su protección.

La zona ha estado sujeta a disturbio antropogénico. Un incendio forestal registrado en 2005, en una porción de la cañada que contiene el arce azucarero, así como en el área aledaña dejó cicatrices de dos metros de largo en árboles de pino. En la región también se extrae madera, principalmente de pino. 


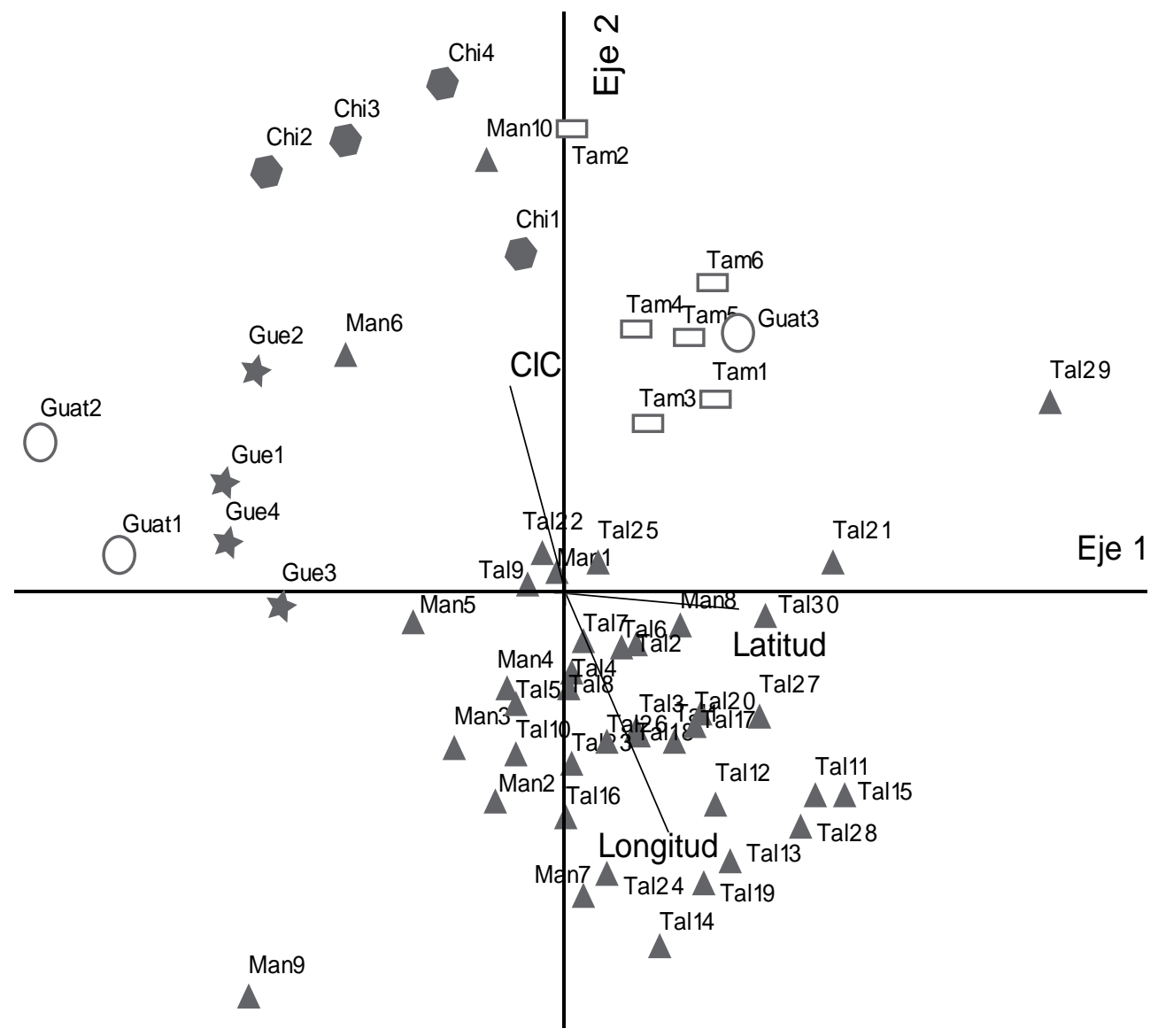

Fig. 3. Diagrama de ordenación NMDS para los ejes 1 y 2, utilizando datos de presenciaausencia de árboles de bosques mesófilos con arce azucarero. Los valores de correlación de las variables ambientales con los ejes se presentan en el cuadro 2 (punto de corte3 desv.). Los nombres de los sitios corresponden a: Tam, Reserva de la Biosfera El Cielo, Tamaulipas; Tal, Talpa de Allende, Jalisco; Man, Reserva de la Biofera Sierra de Manantlán, Jalisco; Gue, barranca El Silencio, Guerrero; Chi, Tenejapa, Chiapas; Guat, Reserva de la Biosfera Sierra de las Minas, Guatemala. Los números corresponden a las parcelas circulares.

\section{DISCUSIÓN}

La riqueza de árboles encontrada en el bosque mesófilo de montaña con arce azucarero en Guerrero fue similar a la hallada en la localidad de Talpa de Allende, Jalisco y superior a la del resto de los sitios donde crece esta planta en México y Guatemala (Vargas-Rodriguez, 2005). La dominancia de la familia Fagaceae en la 
barranca El Silencio fue consistente con la de las otras cinco localidades vegetales conocidas en las que crece la subespecie estudiada y con lo encontrado por Gentry (2001), quien afirma que muchos bosques mesófilos de México y norte de Centroamérica son dominados por miembros de este grupo en altitudes entre 1500 y 2500 m. La dominancia de Asteraceae en la localidad puede estar relacionada con el régimen de perturbación antrópica de la zona, donde los incendios y la tala han sido frecuentes, favoreciendo de tal manera el establecimiento de arbustos de esta familia (Vivar-Evans et al., 2006). Muchos miembros de este grupo conforman los bancos de semillas del suelo del bosque mesófilo, y la apertura de claros del dosel debida al disturbio, favorece la germinación y establecimiento de estas especies (OrtizArrona, 1993).

A pesar de que el área basal total en la zona de estudio fue menor que la de los otros sitios en México y Guatemala, la densidad de árboles fue superior (990 a 2929 individuos por ha-1) (Vargas-Rodriguez, 2005). En el caso del arce azucarero, el área basal registrada representa un valor intermedio dentro de lo encontrado en las otras poblaciones (7.2 a 42.9\%) (Vargas-Rodriguez, 2005). Este patrón puede estar señalando un estado sucesional promovido por disturbio reciente o frecuente, en donde esta última medida es baja y la abundancia alta.

La densidad de plántulas e individuos juveniles de arce es baja o casi nula, lo que pone en riesgo la continuidad de la población. La abundancia de la regeneración natural de la especie en la barranca El Silencio es hasta cuatro veces menor que la encontrada en la Sierra de Manantlán, Talpa de Allende (Jalisco) y en El Cielo (Tamaulipas), sin embargo, supera a las de las poblaciones casi extintas de Tenejapa (Chiapas) (40 individuos) y Las Minas (Guatemala) (27 individuos) (Vargas-Rodriguez, 2005).

En la localidad de Guerrero, el arce azucarero es codominante en el dosel. Esto es consistente con lo encontrado en Estados Unidos y Canadá (Curtis, 1959; Maycock, 1963; Lambert y Maycock, 1968; Roman, 1980; Morrison, 1990; Poulson y Platt, 1996; Dodge, 1997; Beaudet et al., 1999; Arii y Lechowicz, 2002). Sin embargo, la densidad de la especie es menor que las registradas para varios bosques de arce azucarero en E.U. y Canadá, tales como Norberg Creek, 682 árboles ha-1, Wishort Lake, 787 árboles ha-1 (Ontario); Mont St. Hilaire, 248 árboles ha ${ }^{-1}$ (Quebec); Cape Breton Island, 145 árboles ha ${ }^{-1}$ (Nova Scotia) (Greenidge, 1961; Morrison, 1990: Arii y Lechowicz, 2002).

Las propiedades del suelo explican en su mayor parte la variación en la presencia de plantas leñosas en el bosque mesófilo con arce azucarero. Muchos estudios sugieren que los suelos pobres en nutrimentos predisponen la mortalidad de árboles 
(Horsley et al., 2000; Drohan et al., 2002; Bailey et al., 2004; Modry et al., 2004). Igualmente la capacidad de intercambio catiónico, como medida de fertilidad, es importante ya que afecta la retención de los nutrimentos. Este factor también puede prevenir la acidificación edáfica, la cual se ha relacionado con la disminución de la densidad de Acer (Drohan et al., 2002). La deficiencia por magnesio no solamente afecta la supervivencia de los arces jóvenes y adultos, también puede limitar la productividad del bosque (Horsley et al., 2000, Vargas-Rodriguez et al. 2004).

La presencia de especies en bosques mesófilos con arce azucarero muestra un patrón de organización a lo largo de un gradiente geográfico. La distribución de los rodales a lo largo de los ejes de la ordenación señaló una clara distinción de acuerdo con la ubicación geográfica (latitud y longitud) y con respecto a la elevación. Esto indica que los sitios en Tamaulipas y Guatemala son los extremos del gradiente de latitud, y la longitud distingue a los sitios de Jalisco (Fig. 3). Los bosques ubicados a menor elevación estuvieron presentes a mayor latitud y de manera contraria, el bosque de Guatemala, con mayor elevación, se encontró en una latitud menor.

El bosque relicto de arce azucarero en Guerrero posee un elevado número de especies incluidas en alguna categoría de protección oficial. Entre ellas se encuentran árboles de distribución restringida y relicta de los géneros Acer, Magnolia y Abies. La presencia de Abies guatemalensis var. longibracteata constituye el primer registro del taxon para Guerrero.

El elemento endémico está representado en la zona de estudio por la variedad Senna multifoliolata var. metaxi, cuya localidad tipo es cercana al sitio de muestreo (Irwin y Barneby, 1982). Se sugiere la exploración botánica más intensiva y extensiva en la zona, ya que es posible que otras poblaciones de arce azucarero puedan estar en las barrancas de difícil acceso del aledaño ejido Potreritos, donde hay condiciones ambientales similares a las de la barranca El Silencio, propicias para el establecimiento de las mismas.

Los incendios forestales y la extracción de madera en los alrededores de la cañada donde crece el arce azucarero son las principales amenazas para el mantenimiento de la población. La prevención de los primeros es una medida inmediata que puede favorecer a la especie. La creación de una área protegida con reconocimiento federal es también urgente, considerando que la zona se ubica en la cabecera de cuenca hidrológica y cuenta con $30 \%$ de las especies incluidas bajo alguna categoría de protección federal o internacional. La relevancia de la conservación de las poblaciones relictas de Acer saccharum subsp. skutchii ya ha sido reconocida en la mayoría de sus localidades, las cuales han sido incluidas en áreas protegidas de Tamaulipas, Jalisco y Guatemala (Vargas-Rodriguez et al. 2010). 


\section{AGRADECIMIENTOS}

Se agradece el apoyo logístico y financiamiento parcial a las autoridades de la presidencia del municipio de Coahuayutla de José María Izazaga, Guerrero: José Correa, Lorenzo Martínez, Saúl Saucedo y Ricardo Bustos; así como a Antonio Vázquez García y Felipe Monroy Cabrera de la Universidad de Guadalajara e Integración Ciudadana Zapopan, A.C, respectivamente. Los pobladores de los ejidos El Aguacate y Potreritos (Juvenal Herrera, Lorenzo Martínez Nieto y Elías Núñez, entre otros) dieron facilidades para el desarrollo de esta investigación. En el trabajo de campo colaboraron Julia E. Briones Tejeda, Sergio Barrientos Bustamante, Daniel Monroy Rodríguez, Francisco Vargas Aguilar y Antonio Vázquez García; al igual que Silvano Cadena García, Salomón García Chávez, Domingo Ávalos Quintana, Nabor Cárdenas Rodríguez y Saúl Saucedo del municipio Coahuayutla de José María Izazaga. El presente estudio se desarrolló con el apoyo económico del Consejo Nacional de Ciencia y Tecnología (161695) a la autora. Guadalupe Williams-Linera aportó sugerencias valiosas al manuscrito. Luz María González Villarreal y Miguel Cházaro Basáñez ayudaron en la determinación de las plantas.

\section{LITERATURA CITADA}

Anónimo. 1981. Carta climatológica. Carta Guadalajara, escala 1:1000,000. Dirección General de Geografía del Territorio Nacional, Instituto Nacional de Estadística, Geografía e Informática. México, D.F.

Anónimo. 1983a. Carta edafológica E14-A82, escala 1:250,000. Instituto Nacional de Estadística, Geografía e Informática. México, D.F.

Anónimo. 1983b. Carta geológica E14-A82, escala 1:250,000. Instituto Nacional de Estadística, Geografía e Informática. México, D.F.

Anónimo. 1995. Normales climatológicas estándar y provisionales 1961-1990. Comisión Nacional del Agua, Unidad del Servicio Meteorológico Nacional, México, D.F.

Anónimo. 2001. The IUCN Red List Categories and Criteria: Version 3.1. International Union for Conservation of Nature and Natural Resources, Species Survival Commission. IUCN, Gland, Switzerland and Cambridge, UK. 30 pp.

Anónimo. 2002a. Norma Oficial Mexicana NOM-059-ECOL-2001. Protección ambientalespecies nativas de México de flora y fauna silvestres-categorías de riesgo y especificaciones para su inclusión, exclusión o cambio-lista de especies en riesgo. Secretaría de Medio Ambiente y Recursos Naturales, México, D.F.

Anónimo. 2002b. Norma Oficial Mexicana NOM-021-RECNAT-2000, que establece las especificaciones de fertilidad, salinidad y clasificación de suelos. Estudios, muestreos y análisis. Secretaría de Medio Ambiente y Recursos Naturales, México, D.F. 
Anónimo. 2005. Lista roja de flora de Guatemala. Consejo Nacional de Áreas Protegidas (CONAP), Guatemala, Guatemala. 39 pp.

APG (Angiosperm Phylogeny Group). 1998. An ordinal classification for the families of flowering plants. Ann. Missouri Bot. Gard. 85: 531-553.

APG II (Angiosperm Phylogeny Group). 2003. An update of the angiosperm phylogeny group classification for the orders and families of flowering plants: APG II. Bot. J. Linnean Soc. 141: 399-436.

Arii, K. y M. J. Lechowicz. 2002. The influence of overstory trees and abiotic factors on the sapling community in an old-growth Fagus-Acer forest. Ecoscience 9: 386-396.

Bailey, S. W., S. B. Horsley, R. P. Long y R. A. Hallett. 2004. Influence of edaphic factors on sugar maple nutrition and health on the Allegheny Plateau. Soil Sci. Soc. Am. J. 68: 243-252.

Beals, E. W. 1984. Bray-Curtis ordination: an effective strategy for analysis of multivariate ecological data. Adv. Ecol. Res. 14: 1-55.

Beaudet, M., C. Messier, D. Paré, J. Brisson y Y. Bergeron. 1999. Possible mechanisms of sugar maple regeneration failure and replacement by beech in the Boisé-des-Muir old growth forest, Québec. Ecoscience 6: 264-271.

Breedlove, D. E. 1986. Listados florísticos de México. IV. Flora de Chiapas. Instituto de Biología, Universidad Nacional Autónoma de México, México, D.F. 246 pp.

Curtis, J. T. y R. P. McIntosh. 1951. An upland forest continuum in the prairie forest border region of Wisconsin. Ecology 32: 476-496.

Curtis, J. T. 1959. The vegetation of Wisconsin. University of Wisconsin Press. Madison. 657 pp.

Dodge, S. L. 1997. Successional trends in a mixed oak forest on High Mountain, New Jersey. J. Torrey Bot. Soc. 124: 312-317.

Drohan, P. J., S. L. Stout y G. W. Petersen. 2002. Sugar maple (Acer saccharum Marsh.) decline during 1979-1989 in northern Pennsylvania. Forest Ecol. Manag. 170: 1-17.

Faith, D. P., P. R. Minchin y L. Belbin. 1987. Compositional dissimilarity as a robust measure of ecological distance. Vegetatio 69: 57-68.

Figueroa-Rangel, B. y M. Olvera-Vargas. 2000. Regeneration patterns in relation to canopy species composition and site variables in mixed oak forests in Sierra de Manantlán Biosphere Reserve, Mexico. Ecol. Res. 15: 249-261.

Gentry, A. H. 2001. Patrones de diversidad y composición florística en los bosques de las montañas neotropicales. In: Kappelle, M. y A. Brown (eds.). Bosques nublados del neotrópico. Instituto Nacional de Biodiversidad. Santo Domingo de Heredia, Costa Rica. pp. 125-154.

Graham, A. 1999. Late Cretaceous and Cenozoic history of North American Vegetation. Oxford University Press. New York, New York. 370 pp.

Greenidge, K. N. H. 1961. Patterns of distribution of sugar maple, Acer saccharum Marsh., in Northern Cape Breton Island. Am. Midl. Nat. 66: 138-151.

Hernández-Xolocotzi, E., H. Crum, W. B. Fox y A. J. Sharp. 1951. A unique vegetational area in Tamaulipas. Bull. Torrey Bot. Club 78: 458-463.

Horsley, S. B., R. P. Long, S. W. Bailey, R. A. Hallett y T. J. Hall. 2000. Factors associated with the decline disease of sugar maple on the Allegheny Plateau. Can. J. Forest Res. 30: 1365-1378. 
IPNI (International Plant Names Index). 1999. Publicada en internet http://www.ipni.org. (Consultada en 2006).

Irwin, H. S. y R. C. Barneby. 1982. The American Cassiinae. A synoptical revision of Leguminosae tribe Cassieae subtribe Cassiinae in the New World. Mem. New York Bot. Gard. 35: 1-454.

Jardel, E., R. Cuevas, A. Santiago, E. Muñoz y J. Aragón. 1996. Nueva localidad y características de la población de Acer skutchii Rehder en la sierra de Manantlán, Jalisco, México. Acta Bot. Mex. 35: 13-24.

Jiménez-Ramírez, J., K. Vega-Flores, R. Cruz-Durán y J. A. Vázquez-García. 2007. Magnolia guerrerensis (Magnoliaceae) una especie nueva del bosque mesófilo de montaña del estado de Guerrero (México). Bol. Soc. Bot. Méx. 80: 73-76.

Kruskal, J. B. 1964. Nonmetric multidimensional scaling: a numerical method. Psychometrika 29: $115-129$.

Lambert, J. D. H. y P. F. Maycock. 1968. The ecology of terricolous lichens of the northern Conifer-Hardwood forests of eastern Canada. Can. J. Bot. 46: 1043-1078.

Magurran, A. E. 1988. Ecological diversity and its measurement. Princeton University. Princeton, New Jersey. 192 pp.

Martin, P. S. y B. E. Harrell. 1957. The Pleistocene history of temperate biotas in Mexico and eastern United States. Ecology 38: 468-480.

Mather, P. M. 1976. Computational methods of multivariate analysis in physical geography. J. Wiley \& Sons. Londres. 532 pp.

Maycock, P. F. 1963. The phytosociology of the deciduous forest of extreme southern Ontario. Can. J. Bot. 41: 379-438.

McCune, B. y M. J. Mefford. 1999. PC-ORD. Multivariate analysis of ecological data: version 4.0. MjM Software. Gleneden Beach, Oregon.

McCune, B. y J. B. Grace. 2002. Analysis of ecological communities. MjM Software Design. Gleneden Beach, Oregon.

Medinilla-Sánchez, O. E. 1999. Estudio florístico de los bosques con dominancia de especies del género Pinus en la microcuenca del río Colorado, Río Hondo, Zacapa. Tesis de licenciatura. Universidad San Carlos de Guatemala. Guatemala. 136 pp.

Minchin, P. R. 1987. An evaluation of the relative robustness of techniques for ecological ordination. Vegetatio 69: 89-107.

Modry, M., D. Hubeny y K. Rejsek. 2004. Differential response of naturally regenerated European shade tolerant tree species to soil type and light availability. Forest Ecol. Manag. 188: 185-195.

Morrison, I. K. 1990. Organic matter and mineral distribution in an old-growth Acer saccharum forest near the northern limit of its range. Can. J. Forest Res. 20: 1332-1342.

Murray, A. E. Jr. 1975. North American maples. Kalmia 7: 1-19.

Olvera-Vargas, M., S. Moreno y B. Figueroa-Rangel. 1996. Sitios permanentes para la investigación silvícola, manual para su establecimiento. Universidad de Guadalajara. Guadalajara. 56 pp.

Ortiz-Arrona, C. I. 1993. Banco de semillas en el suelo del bosque mesófilo de montaña en la Estación Científica Las Joyas. Tesis profesional. Facultad de Ciencias Biológicas, Universidad de Guadalajara. Guadalajara. 90 pp. 
Poulson, T. L. y W. J. Platt. 1996. Replacement patterns of beech and sugar maple in Warren Woods, Michigan. Ecology 77: 1234-1253.

Qian, H. y R. E. Ricklefs. 2000. Large-scale processes and the Asian bias in species diversity of temperate plants. Nature 407: 180-182.

Rehder, A. 1936. A new species of Acer from Guatemala. J. Arnold Arboretum 17: 350351.

Roman, J. R. 1980. Vegetation-environment relations in virgin, middle elevation forests in the Adirondack Mountains, New York. Tesis de doctorado. State University of New York. Syracuse, New York. 477 pp.

Vargas-Rodriguez, Y. L. 2005. Ecology of disjunct cloud forest sugar maple populations (Acer saccharum subsp. skutchii) in North and Central America. Tesis de maestría en ciencias. Louisiana State University. Baton Rouge, Louisiana. 130 pp.

Vargas-Rodriguez, Y. L., J. A. Vázquez-García y W. Platt. 2004(2005). Gradientes ambientales en el establecimiento de poblaciones relictas de Acer saccharum subsp. skutchii y Podocarpus reichei en el occidente de México. Ibugana 12: 35-41.

Vargas-Rodriguez, Y. L., W. Platt, J. A. Vázquez-García y G. Boquin. 2010. Selecting relict montane cloud forests for conservation priorities: the case of western Mexico. Nat. Area J. 30: 156-173.

Vázquez-García, J. A., Y. L. Vargas-Rodriguez y F. Aragón. 2000. Descubrimiento de un bosque de Acer-Podocarpus-Abies en el municipio de Talpa de Allende, Jalisco, México. Ibugana 7: 159-183.

Vivar-Evans, S., V. L. Barradas, M. E. Sánchez-Coronado, A. G. de Buen y A. Orozco-Segovia. 2006. Ecophysiology of seed germination of wild Dahlia coccinea (Asteraceae) in a spatially heterogeneous fire-prone habitat. Acta Oecol. 29: 187-195. 